\title{
Nonlinear rock and roll - Modeling and control of parametric resonances in wave energy devices
}

\author{
Josh Davidson* Tamas Kalmar-Nagy ** Giuseppe Giorgi * \\ John V. Ringwood* \\ * Centre for Ocean Energy Research, Maynooth University, Ireland \\ (e-mail: josh.davidson@mu.ie). \\ ** Dept Fluid Dynamics, Budapest University of Technology and \\ Economics, Hungary (e-mail: kalmarnagy@ara.bme.hu)
}

Keywords: Five to ten keywords, preferably chosen from the IFAC keyword list.

\section{INTRODUCTION}

The concept of resonance is well known in the study of wave energy converters (WECs), with the natural frequency of WECs typically designed to resonate with the external excitation provided by the input wave field. Parametric resonance, on the other hand, has received very little attention, likely due to the complexity of the models required to capture this nonlinear phenomenom, compared to the traditional linear/frequency domain models favoured in WEC research and analysis. This presentation examines the modeling methods available for simulating, analysing and controlling parametric resonances in wave energy devices. The traditional linear hydrodynamic models are discussed and contrasted against nonlinear hydrodynamic modelling approaches, in terms of model fidelity and computational requirements. The effect of the mooring system dynamics on the parametric excitation of the WEC system is discussed and modelling methods reviewed. Finally, the effect of the WEC's power take-off (PTO) system on extracting energy from the WEC and controlling the occurance of parametric resonance is detailed.

\section{PARAMETRIC RESONANCE IN WECS}

Parametric resonance is a phemonenon caused by the timevarying changes in the parameters of a system (Fossen and Nijmeijer (2011)). While resonance causes the oscillations of a system to grow linearly with time (until damping limits further growth), parametric resonance causes an exponential increase in oscillation amplitude, and can often be unexpected since it is a nonlinear phenomenom not predicted by linear analyses. Parametric resonance has been observed and studied in floating bodies, dating back to the work of Froude (1861) who described that large roll motions occur when a ship's roll natural period is twice the heave/pitch natural period. This parametric coupling between modes of motion has been investigated in offshore engineering fields, where parametric resonance is considered an unwanted problem (with container ships losing cargo due to large parametric roll motions for example),

\footnotetext{
* This paper is based upon work supported by Science Foundation Ireland under Grant No. 13/IA/1886.
}

and research focussing on suppression and stabilisation of parametric pitch/roll.

Likewise, parametric resonance has been observed to induce large amplitude pitch/roll motions for WECs, however very little investigation has been undertaken. Babarit et al. (2009) assessed the potential of an advanced numerical model in capturing the nonlinear hydrodynamics of a pitching type WEC, as compared against physical wave tank experiments. Of interest, for wave frequencies close to half the WEC roll natural frequency, parametric roll was observed in the experiments, which was only predicted by advanced nonlinear hydrodynamic models, but not the traditional linear ones. Tarrant and Meskell (2016) investigated the parametric resonance in a 2-body self-reacting WEC, due to the varying centre of mass as the bodies translate relative to each other. Gomes et al. (2017) investigated experimentally how additional damping may surpress the parametric resonance in a spar-bouy type oscillating water column (OWC) WEC. In these studies, the effect of parametric resonance was detrimental to the WEC performance, reducing the power capture as energy was parametrically transferred from the primary mode of motion into other modes.

However, unlike most other offshore applications, the large amplitude motions caused by parametric resonance could also be considered as beneficial for certain types of WECs designed to extract energy from these modes. Olvera et al. (2001) first proposes utilising parametric resonance, for the case of an OWC, whereby the parametric excitation caused by varying the volume of the OWC chamber is investigated (Olvera et al. (2007)). Orazov (2011) and Diamond et al. (2015) propose a class of WEC that utilises parametric resonance by a mass-modulation scheme, whereby the mass of a WEC element is varied in time through control that either varied the added mass or takes on water. In addition to stabilisation, Galeazzi and Pettersen (2012) outlines using control to induce parametric resonance in floating bodies, citing wave energy as one area where this application may be desirable. This topic was also recently debated at the 2017 Maynooth Wave Energy Workshop, during an open forum discussion session (Clement (2017)). 
Parametric resonance may therefore be considered either detrimental or beneficial for different types of WECs, and control systems may need to be designed to either mitigate or induce this effect.

\section{WEC FORCES AND CONTROL}

The forces on the WEC comprise: the force from the fluid, the force from the mooring system and the force from the PTO (in addition to gravity). The modelling of each of these forces and their role in the parametric resonance of a WEC is discussed in this presentation.

\subsection{Fluid force}

A range of methods have been developed to model the fluid-structure interaction, details of which can be found in Folley (2016). This presentation discusses the traditional linear hydrodynamic modelling techniques, classically used due to computational restrictions in solving the full nonlinear Navier-Stokes set of equations, but which are unable to capture the nonlinear phemonena of parametric resonance. Modifyig these linear modeling techniques to be cast into the form of the classic Mathieu-Hill equation for studying parametric resonance is then discussed. Next, nonlinear potential flow methods are discussed and example results from Giorgi and Ringwood (2018) presented. The role of viscous damping is then discussed, along with methods to model this effect. Finally, the high-fidelity / computationally expensive approaches offered by CFD are explored.

\subsection{Mooring force}

The role in which the mooring system can serve in either inducing or mitigating parametric resonance, and methods to model these mooring effects, is discussed in this presentation. For example, mooring forces can introduce cross-coupling between the heave and pitch/roll modes of motion, depending on where the mooring line(s) is attached to the WEC. Also, drag forces on the mooring lines can present a large source of energy dissipation from the WEC. These and other effects are discussed in detail, drawing upon the indepth review of the mathematical modelling of mooring systems for WECs given in Davidson and Ringwood (2017).

\subsection{PTO force}

The PTO system converts the mechanical energy in the WEC into other forms of energy (electric, hydraulic, etc.) via the PTO force. The PTO force can act in a chosen degree of freedom with a specified amplitude and is therefore a useful control input. The role in which the PTO can be used to control parametric resonance in a WEC is explored in this presentation, considering traditional energy maximising control methods for WECs Ringwood et al. (2014), as well as other methods such as nonlinear energy sinks Vakakis et al. (2008).

\section{CONCLUSION}

A range of mathematical models, spanning the 'model fidelity-computational requirement' plane, are available for the analysis and control of parametric resonance in WECs. Low fidelity models are useful for quick analyses of the stability properties, where as higher fidelity, more computationally heavy, models are useful for evaluation purposes or system identification of lower fidelity models.

\section{REFERENCES}

Babarit, A., Mouslim, H., Clément, A., and LaporteWeywada, P. (2009). On the numerical modelling of the nonlinear behaviour of a wave energy converter. In Proceedings of the 28th International Conference on Offshore Mechanics \& Arctic Engineering.

Clement, A. (2017). Instabilities of wecs: Good news? bad news? Available at: www.eeng.nuim.ie/coer/pastevents/2017-maynooth-wave-energy-workshop/.

Davidson, J. and Ringwood, J.V. (2017). Mathematical modelling of mooring systems for wave energy convertersa review. Energies, 10(5), 666.

Diamond, C.A., Judge, C.Q., Orazov, B., Savaş, Ö., et al. (2015). Mass-modulation schemes for a class of wave energy converters: Experiments, models, and efficacy. Ocean Engineering, 104, 452-468.

Folley, M. (2016). Numerical Modelling of Wave Energy Converters: State-of-the-Art Techniques for Single Devices and Arrays. Academic Press.

Fossen, T. and Nijmeijer, H. (2011). Parametric resonance in dynamical systems. Springer.

Froude, W. (1861). On the rolling of ships. Institution of Naval Architects.

Galeazzi, R. and Pettersen, K.Y. (2012). Parametric resonance in dynamical systems, chapter Controlling Parametric Resonance: Induction and Stabilization of Unstable Motions, 305-327. Springer.

Giorgi, G. and Ringwood, J.V. (2018). Analytical formulation of nonlinear froude-krylov forces for pitching point absorbers. In Submitted to the 37th International Conference on Ocean, Offshore and Arctic Engineering.

Gomes, R., Malvar Ferreira, J., Ribeiro e Silva, S., Henriques, J., and Gato, L. (2017). An experimental study on the reduction of the dynamic instability in the oscillating water column spar buoy. In Proceedings of the 12th European Wave and Tidal Energy Conference.

Olvera, A., Prado, E., and Czitrom, S. (2001). Performance improvement of owc systems by parametric resonance. In Proceedings of the 4 th European Wave Energy Conference.

Olvera, A., Prado, E., and Czitrom, S. (2007). Parametric resonance in an oscillating water column. Journal of Engineering Mathematics, 57(1), 1-21.

Orazov, B. (2011). A novel excitation scheme for an ocean wave energy converter. Ph.D. thesis.

Ringwood, J.V., Bacelli, G., and Fusco, F. (2014). Energymaximizing control of wave-energy converters: The development of control system technology to optimize their operation. IEEE Control Systems, 34(5), 30-55.

Tarrant, K. and Meskell, C. (2016). Investigation on parametrically excited motions of point absorbers in regular waves. Ocean Engineering, 111, 67-81.

Vakakis, A.F., Gendelman, O.V., Bergman, L.A., McFarland, D.M., Kerschen, G., and Lee, Y.S. (2008). Nonlinear Targeted Energy Transfer in Mechanical and Structural Systems: I and II. Springer-Verlag, Berlin and New York. 